\title{
CARACTERIZAÇÃO QUÍMICA E EFEITO DO ÓLEO ESSENCIAL DE ERVA-DE-SANTA-MARIA SOBRE O ÁCARO-RAJADO DE MORANGUEIRO'
}

\author{
JOÃO PAULO PEREIRA PAES ${ }^{2}$, VANDO MIOSSI RONDELLI ${ }^{3}$, ADILSON VIDAL COSTA $^{4}$, \\ ULYSSES RODRIGUES VIANNA ${ }^{5}$, VAGNER TEBALDI DE QUEIROZ ${ }^{6}$
}

RESUMO- O ácaro-rajado, Tetranychus urticae Koch (1836) (Acari: Tetranychidae), é a principal praga na cultura do morangueiro. O objetivo deste trabalho foi a caracterização química do óleo essencial de Chenopodium ambrosioides L. e a avaliação de seus efeitos sobre o ácaro-rajado. Os compostos do óleo essencial foram identificados pelas análises de CG-DIC e CG-EM. A mortalidade e a oviposição foram avaliadas após a pulverização do óleo $\left(2,5 ; 5,0 ; 7,5\right.$ e 10,0\% v v $\left.\mathrm{v}^{-1}\right)$ sobre fêmeas do ácaro-rajado. O rendimento do óleo essencial foi de $0,3 \%$, e os compostos identificados foram (Z)-ascaridol (87\%), (E)-ascaridol (5,04\%), p-cimeno $(4,83 \%), \alpha$ - terpineno $(1,24 \%)$ e piperitona $(0,7 \%)$. Os maiores valores para mortalidade e redução de oviposição observados foram $85 \%$ e $69 \%$, respectivamente. O óleo essencial de C. ambrosioides é uma opção promissora para o manejo do ácaro-rajado.

Termos para Indexação: óleo volátil; Chenopodium ambrosioides; bioacaricida; Tetranychus urticae.

\section{CHEMICAL CHARACTERIZATION AND EFFECT OF ESSENTIAL OIL FROM MEXICAN-TEA ON TWOSPOTTED SPIDER MITE}

\begin{abstract}
The twospotted spider mite, Tetranychus urticae Koch (1836) (Acari: Tetranychidae), is the major pest in strawberry. The objective of this study was the chemical characterization of the essential oil of Chenopodium ambrosioides L. and the evaluation of their effects on twospotted spider mite. The compounds of the essential oil were identified by GC/FID and GC/MS analysis. The mortality and oviposition were evaluated after spraying the oil $\left(2.5,5.0,7.5\right.$ and $\left.10.0 \% \mathrm{v} \mathrm{v}^{-1}\right)$ on twospotted spider mite females. The yield of the essential oil was of $0.3 \%$ and the compounds identified were (Z)-ascaridol (87\%), (E)-ascaridol $(5.04 \%)$, p-cymeno $(4.83 \%), \alpha$-terpinene $(1.24 \%)$ and piperitone $(0.7 \%)$. The highest values for mortality and reduction of oviposition observed were $85 \%$ and $69 \%$, respectively. C. ambrosioides essential oil is a promising option for the management of twospotted spider mite.
\end{abstract}

Index terms: volatile oil; Chenopodium ambrosioides; bioacaricide; Tetranychus urticae.

\footnotetext{
'(Trabalho 057-14). Recebido em: 13-02-2014. Aceito para publicação em: 27-01-2015.

2Engenheiro Agrônomo, Mestrado em Produção Vegetal” Departamento de Produção Vegetal, Universidade Federal do Espírito Santo, Centro de Ciências Agrárias, Alto Universitário, s/nº, Guararema, 29500-000, Alegre, Espírito Santo, Brasil. E-mail: joaopauloppaes@ hotmail.com

${ }^{3}$ Engenheiro Agrônomo, Doutorado em Entomologia Agrícola, Departamento de Agronomia e Entomologia, Universidade Federal Rural de Pernambuco, Avenida Dom Manoel de Medeiros, s/nº, Dois Irmãos, 52171-900, Recife, Pernambuco, Brasil. E-mail:rondellimiossi@hotmail.com

${ }^{4}$ Químico, Doutorado em Química, Departamento de Química e Física, CCA-UFES. E-mail: avcosta@hotmail.com (autor para correspondência).

${ }^{5}$ Engenheiro Agrônomo, Doutorado em Entomologia, Departamento de Produção Vegetal, CCA-UFES. E-mail: ulyssesvianna@ hotmail.com

${ }^{6}$ Químico, Doutorado em Bioquímica Agrícola, Departamento de Química e Física, CCA-UFES. E-mail: vagnertq@gmail.com
} 


\section{INTRODUÇÃO}

O morangueiro (Fragaria x ananassa Duch) é resultado de uma hibridação interespecífica de duas espécies selvagens do mesmo. Trata-se de uma das mais importantes pequenas frutas do mundo, no cultivo da qual muitas famílias de pequenos e médios produtores estão envolvidas (FERLA et al., 2007; FERLA et al., 2011). No entanto, essa cultura é suscetível a pragas e doenças, sendo algumas de difícil manejo e, por isso, causam muitas perdas (HENZ, 2010).

O ácaro-rajado, Tetranychus urticae Koch (1836) (Acari: Tetranychidae), apresenta grande relevância agrícola em função das perdas ocasionadas pela sua ocorrência em diferentes sistemas de cultivo, entre os quais frutas, vegetais, plantas ornamentais e medicinais (HAN et al., 2011). A principal praga na cultura do morangueiro é o ácaro-rajado, sendo o período ideal para o seu crescimento elevadas temperaturas e baixas precipitações (FADINI; VENZON, 2004). A infestação na época de produção de mudas pode provocar atraso no desenvolvimento das plantas, enfraquecimento e diminuição na emissão dos estolões com consequente diminuição do número de mudas produzidas (MORAES; FLECHTMANN, 2008).

O controle de T. urticae pode ser feito com produtos à base de nim (azadiractina), ácaros predadores e fungo entomopatogênico (WEKESA et al., 2011; SCHLESENER et al., 2013). Além disso, o controle do ácaro-rajado também pode ser efetuado por meio da aplicação de acaricidas sintéticos (SATO et al., 2002). Entretanto, a resistência desta praga a tais produtos tem estimulado a busca por novos métodos de controle, entre os quais, a implantação de sistemas de manejo fitossanitário, utilizando espécies vegetais com propriedades inseticidas. Nos últimos anos, diferentes trabalhos de pesquisa relataram o potencial de óleos essenciais de plantas (MIRESMAILLI et al., 2006; CALMASUR et al., 2006; PONTES et al., 2007a) e extratos vegetais (SHI et al., 2006; VIEIRA et al., 2006; BRITO et al., 2006) para o controle de ácaros. Substâncias isoladas a partir de produtos naturais também têm sido utilizadas como modelos para a semissíntese de agentes acaricidas (TSUKAMOTO et al., 1997).

A erva-de-santa-maria, Chenopodium ambrosioides L. (Chenopodiaceae), é uma espécie vegetal promissora para utilização em sistemas de manejo fitossanitário de pragas, uma vez que possui atividade repelente e inseticida sobre diversas famílias de insetos (PROCÓPIO et al., 2003; TAVARES;VENDRAMIM, 2005; GIRÃO FILHO et al., 2014).

Neste contexto, o objetivo deste trabalho foi caracterizar químicamente o óleo essencial de $C$. ambrosioides e avaliar a mortalidade causada em $T$. urticae, em laboratório.

\section{MATERIAL E MÉTODOS}

\section{Coleta do material vegetal}

As partes aéreas de C. ambrosioides foram coletadas no período matutino, em novembro de 2011, a partir de plantas cultivadas em casa de vegetação, localizada no município de Alegre, Estado do Espírito Santo, nas coordenadas de 20 44' 49" de latitude $\mathrm{S}, 41^{\circ} 27^{\prime} 58^{\prime}$ " de longitude $\mathrm{W}$ e altitude de

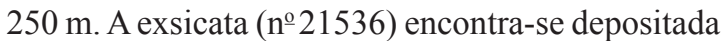
no herbário da Universidade Federal do Espírito Santo-(VIES)-Subcuradoria Câmpus de Alegre.

\section{Extração do óleo essencial}

O óleo essencial foi obtido por hidrodestilação em laboratório. Folhas da planta fresca (cerca de 100 g) foram transferidas para um balão, que foi acoplado ao aparelho Clevenger e este ao condensador. A hidrodestilação foi mantida após ebulição da água, por $3 \mathrm{~h}$. Após obtenção de aproximadamente 500 $\mathrm{mL}$ de hidrolato, foi realizada extração, utilizando o pentano como solvente. Foram realizadas cinco extrações com $40 \mathrm{~mL}$ de pentano, recolhendo a fase orgânica. Nesta, foi vertida uma quantidade em excesso de sulfato de sódio anidro para retirada de água da amostra, procedendo a sua filtração. O filtrado foi levado ao evaporador rotativo para a obtenção do óleo essencial por remoção do solvente (CASTRO et al., 2004; COSTA et al., 2013; PINHEIRO et al., 2013). O óleo essencial de C. ambrosioides foi obtido com rendimento de $0,3 \%\left(\mathrm{~m} \mathrm{v}^{-1}\right)$.

\section{Caracterização química}

A identificação dos compostos foi realizada por cromatografia gasosa acoplada ao espectrômetro de massas (CG-EM), em equipamento com detector seletivo de massa, modelo QP-PLUS-2010 (SHIMADZU). A coluna cromatográfica utilizada foi do tipo capilar de sílica fundida com fase estacionária Rtx-5MS, de $30 \mathrm{~m}$ de comprimento e $0,25 \mathrm{~mm}$ de diâmetro interno, utilizando hélio como gás de arraste. As temperaturas foram de $220^{\circ} \mathrm{C}$ no injetor e $300{ }^{\circ} \mathrm{C}$ no detector. A temperatura inicial da coluna foi de $60{ }^{\circ} \mathrm{C}$, sendo programada para ter acréscimos de $3{ }^{\circ} \mathrm{C}$ a cada minuto, até atingir a temperatura máxima de $240{ }^{\circ} \mathrm{C}$ (CASTRO et al.; 2004, COSTA et al., 2013; PINHEIRO et al., 2013). A identificação dos compostos foi obtida por comparações dos 
espectros de massas com os existentes na biblioteca NIST, com a literatura e pelo índice de Kovat's (ADAMS, 2007).

A quantificação dos constituintes químicos do óleo essencial foi realizada por cromatografia em fase gasosa, em equipamento SHIMADZU GC-2010 Plus, equipado com detector de ionização de chama (CG-DIC). O gás de arraste utilizado foi o nitrogênio e coluna capilar Rtx-5MS, $30 \mathrm{~m}$ de comprimento e $0,25 \mathrm{~mm}$ de diâmetro interno. As temperaturas do injetor e do detector foram fixadas em 240 e $250{ }^{\circ} \mathrm{C}$, respectivamente. A programação de temperatura no forno foi a mesma utilizada nas análises por CGEM. A quantidade de $10 \mathrm{mg}$ das amostras foi diluída em $1 \mathrm{~mL}$ de diclorometano, sendo injetado $1 \mu \mathrm{L}$ da mistura (CASTRO et al., 2004; COSTA et al., 2013; PINHEIRO et al., 2013).

\section{Criação de T. urticae}

Os ácaros usados nos experimentos foram provenientes da criação-estoque mantida no Núcleo de Desenvolvimento Científico e Tecnológico em Manejo Fitossanitário de pragas e doenças (NUDEMAFI), coletados no campo, em janeiro de 2008, em folhas de morangueiro, e transferidos para plantas de feijão-de-porco, Canavalia ensiformis L. (Fabaceae), de acordo com a metodologia desenvolvida por Brito et al. (2006).

\section{Bioensaio}

Discos de folha de feijão-de-porco (4 cm de diâmetro), proveniente de um cultivo onde não houve aplicação de agrotóxicos ou outro meio de controle de pragas, foram acondicionados em placas de Petri $(9,0 \times 1,3 \mathrm{~cm})$. No interior das placas, foi colocado algodão úmido para promover a formação de uma arena e evitar a fuga dos ácaros. Cada disco foi infestado com 15 fêmeas adultas de T. urticae (repetição) de até 24 h, obtidas da criação-estoque do NUDEMAFI.

Pulverizaram-se os discos foliares com solução do óleo essencial, utilizando-se de $6 \mathrm{~mL}$ por repetição, em torre de Potter calibrada à pressão de $15 \mathrm{lb} \mathrm{pol}^{-2}$, tendo-se depositado um volume de 1,62 $\mathrm{mg} \mathrm{cm}{ }^{-2}$ (RONDELLI et al., 2011). As concentrações do óleo essencial utilizadas foram de 2,5; 5,0; 7,5 e $10,0 \% \mathrm{v} \mathrm{v}^{-1}$, solubilizadas em espalhante adesivo Tween ${ }^{\circledR} 80\left(0,05 \% \mathrm{~V} \mathrm{v}^{-1}\right)$. Na testemunha, utilizouse solução de água destilada e espalhante adesivo Tween ${ }^{\circledR} 80\left(0,05 \% \mathrm{~V} \mathrm{v}^{-1}\right)$. Os parâmetros avaliados, após a pulverização, foram mortalidade (24; 48 e $72 \mathrm{~h}$ ) e número total de ovos depositados ( $24 \mathrm{~h}) . \mathrm{O}$ bioensaio foi conduzido à temperatura de $25 \pm 1{ }^{\circ} \mathrm{C}$, umidade relativa $70 \pm 10 \%$ e fotofase de $12 \mathrm{~h}$.
Foi utilizado o delineamento experimental inteiramente casualizado, com 10 repetições, contendo 15 fêmeas adultas de $T$. urticae em cada repetição. Os dados foram submetidos à análise de regressão, ao nível de 5\% de probabilidade.

\section{RESULTADOS E DISCUSSÃO}

\section{Caracterização química}

O óleo essencial de C. ambrosioides foi obtido com rendimento de $0,3 \%$. Estudos realizados com amostras de C. ambrosioides coletadas em Ruanda, Nigéria e Índia relatam valores de rendimento para o óleo essencial variando entre 0,06 e 0,3\% (MUHAYIMANA et al., 1998; ONOCHA et al., 1999; GUPTA et al., 2002). Entre plantas da mesma espécie, as variações no teor de óleo essencial podem ser atribuídas, principalmente, a diferenças de época de colheita, tipo de solo e clima da região (BURT, 2004).

A análise por CG-FID e CG-EM possibilitou a quantificação de $98,8 \%$ dos constituintes químicos presentes no óleo essencial das folhas de erva-desanta-maria (Tabela 1). Os principais compostos identificados foram (Z)-ascaridol (87\%), (E)ascaridol (5,04\%), p-cimeno (4,83\%), $\alpha$-terpineno $(1,24 \%)$ e piperitona $(0,7 \%)$. Observa-se ainda que os compostos presentes neste óleo divergem em relação a seus teores. Assim como descrito por Cavalli et al. (2004), o (Z)-ascaridol representa o principal constituinte químico encontrado neste estudo. Onocha et al. (1999) e Gupta et al. (2002) descrevem o $\alpha$-terpineno como composto majoritário, enquanto este componente se apresentou com teor de 1,24\% no presente trabalho. Embora Tapondjou et al. (2002) relatem o p-cymeno com 50\% de ocorrência e como constituinte principal no óleo essencial de $C$. ambrosioides, neste estudo, o teor encontrado para este composto foi de $4,83 \%$.

Uma vez que o óleo essencial de $C$. ambrosioides utilizado nos trabalhos sumarizados na Tabela 1 foi obtido a partir de amostras coletadas em diferentes regiões (Índia, Camarões, Nigéria e França), provavelmente, as diferenças observadas na constituição química destes estejam relacionadas com a época de colheita, tipo de clima e solo.

\section{Bioensaio}

A mortalidade das fêmeas de T. urticae aumentou em função da concentração de óleo essencial de erva-de-santa-maria e em função dos tempos de avaliação, tendo os dados se ajustado ao modelo de regressão linear (Figura 1). Na testemunha, a porcentagem de mortalidade observada foi $5 \%$. Na 
avaliação de 24 h, a concentração do óleo essencial a $10 \% \mathrm{v} \mathrm{v}^{-1}$ foi a que ocasionou maior mortalidade, aproximadamente $60 \%$ (Figura 1 ).

Nas avaliações de 48 e 72 h, os maiores valores observados para mortalidade também foram na concentração de $10 \% \mathrm{v} \mathrm{v}^{-1}$, com 70 e $85 \%$, respectivamente, o que representa um aumento na mortalidade em relação à avaliação de $24 \mathrm{~h}$. O aumento da mortalidade da praga também foi observado em todos os outros tratamentos, inclusive na testemunha, onde a mortalidade foi de aproximadamente $23 \%$ na avaliação de 72 h (Figura 1). Dessa forma, em todas as avaliações, as regressões apresentaram a mesma tendência, pois os coeficientes angulares das retas foram próximos.

As altas mortalidades observadas nas avaliações de 48 e $72 \mathrm{~h}$ na testemunha (14 e 23\%, respectivamente), provavelmente, foram devido à mortalidade natural da praga, uma vez que a mortalidade em todos os tratamentos aumentou proporcionalmente. Desta forma, fica evidente que o óleo essencial causou mortalidade da praga apenas nas primeiras $24 \mathrm{~h}$ após a pulverização, pelo fato de o óleo essencial ser extremamente volátil, sendo possível, nas condições em que esse experimento foi conduzido, apenas a ação aguda sobre o ácarorajado. Chiasson et al. (2004), avaliando o formulado UDA-245 (25\% de ingrediente ativo), à base do óleo essencial de C. ambrosioides na concentração de $0,5 \%$, não observaram efeito residual quando o ácaro-rajado foi inoculado sobre os discos foliares previamente pulverizados uma hora antes. A penetração do óleo essencial, provavelmente, ocorreu via tegumento, pois a composição lipídica da cutícula do ácaro favorece sua absorção (CRESPO et al., 2002).

O efeito acaricida do óleo essencial de erva-de-santa-maria pode ser atribuído aos monoterpenos presentes nesse óleo, que inibem a enzima acetilcolinesterase e o citrocromo P450 (DE-OLIVEIRA et al., 1997; RYAN;BYRNE, 1988). Além disso, o monoterpeno ascaridol, que compõe $92 \%$ do óleo essencial de C. ambrosioides (Tabela 1), pode ter apresentado efeito tóxico sobre as mitocôndrias, por inibir a cadeia de transporte de elétrons (MONZOTE et al., 2009).

O produto UDA-245, na concentração de $0,5 \%$ em pulverização tópica, proporcionou mortalidade de 94,7\% de fêmeas adultas de T. urticae. Esse formulado também apresentou atividade ovicida, contudo com alta porcentagem de eclosão de larvas (81,1\%) (CHIASSON et al., 2004). Além disso, este mesmo produto (concentração de $0,5 \%$ ) também apresentou suave efeito tóxico ao predador
Orius insidiosus (Say) e ao parasitoide Aphidius colemani Viereck (BOSTANIAN et al., 2005).

Contudo, o produto QRD 400 (concentrado emocionável a $25 \%$ de ingrediente ativo), formulado a partir de óleo essencial de C. ambrosioides, quando pulverizado sobre Planococcus citri (Risso) e Frankliniella occidentalis (Pergande), em casa de vegetação, proporcionou baixa taxa de mortalidade sobre essas pragas (CLOYD; CHIASSON, 2007).

Jaramillo et al. (2012), estudando o óleo essencial de erva-de-santa-maria na concentração de $0,5 \mathrm{~mL} \mathrm{~L}^{-1}$ de ar, obtiveram $100 \%$ de mortalidade de adultos de Sitophilus zeamais (Mots.). Além disso, o pó de folhas, flores e frutos de erva-de-santa-

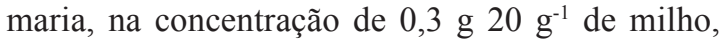
foi suficiente para causar $100 \%$ de mortalidade de adultos de $S$. zeamais. Também foi observado que nenhum adulto emergiu desse tratamento na concentração de $0,6 \mathrm{~g} \mathrm{~g}^{-1}$ de milho (PROCÓPIO et al., 2003). Em outro estudo, o pó de folha de erva-desanta-maria foi menos tóxico para $S$. zeamais, sendo

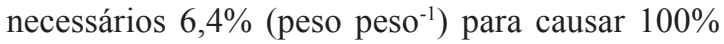
de mortalidade. No entanto, para Callosobruchus chinensis (L.), foi necessária a concentração de

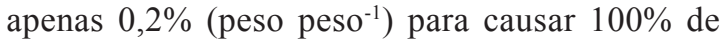
mortalidade (TAPONDJOU et al., 2002).

O número de ovos depositados por T. urticae diminuiu em função do aumento da concentração de óleo essencial de erva-de-santa-maria, tendo os dados se ajustado ao modelo de regressão linear (Figura 2). Na testemunha, foi observada média de oviposição de 58 ovos, enquanto na concentração de $10 \%$ do óleo essencial foram verificados 18 ovos, o que representa uma redução de 69\%. Menos ovos foram observados na maior concentração do óleo essencial porque havia menos fêmeas vivas nesta concentração (Figura 1). Contudo, a ação tóxica do óleo essencial também pode ter tido efeito de deterrência de oviposição sobre os ácaros que sobreviveram. Estes eventos contribuem para que ocorra redução populacional da praga na próxima geração. A repelência é outro efeito que pode ter ocorrido com os ácaros, pois o óleo essencial de fruto de Protium heptaphyllum (Aubl.) Marchand (Burseraceae) causou repelência do ácaro-rajado, reduzindo a alimentação e, consequentemente, a fecundidade (PONTES et al., 2007b). 

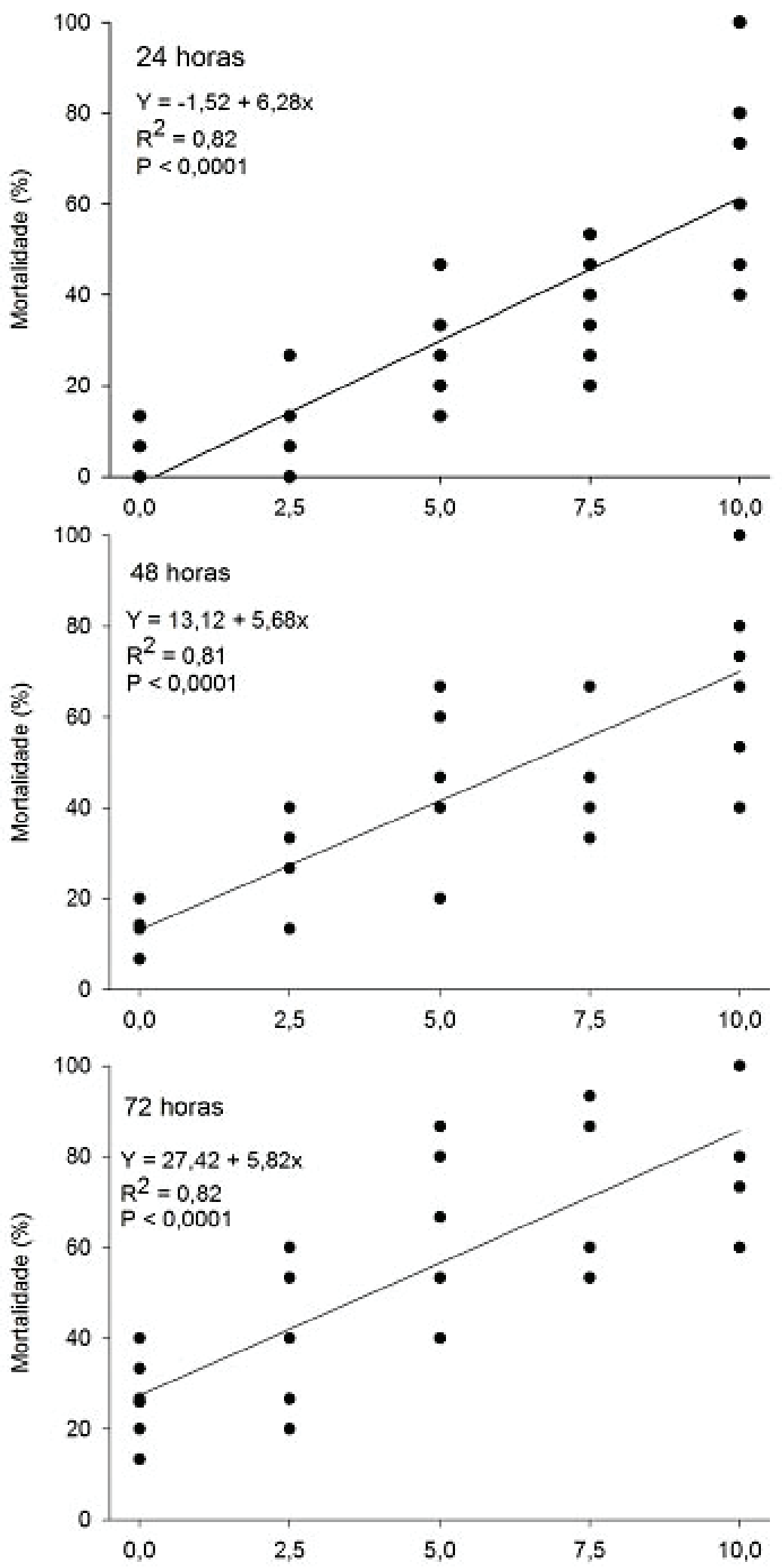

FIGURA 1 - Mortalidade de fêmeas Tetranychus urticae tratadas com óleo essencial de erva-de-santa-maria, Chenopodium ambrosioides, a diferentes concentrações $\left(\mathrm{v} \mathrm{v}^{-1}\right)$ após 24, 48 e $72 \mathrm{~h}$. 
TABELA 1 - Composição química do óleo essencial de erva-de-santa-maria, Chenopodium ambrosioides, representado pelo índice de Kovats calculado e tabelado e porcentagem relativa de cada substância do óleo estudado e de outros estudos.

\begin{tabular}{ccccccccc}
\hline & \multicolumn{3}{c}{ Índice de Kovats } & \multicolumn{5}{c}{ Porcentagem relativa } \\
\cline { 2 - 7 } Pico & \multirow{2}{*}{ Cal. } & \multirow{2}{*}{ Tab. } & \multirow{2}{*}{ Composto } & \multicolumn{2}{c}{ Óleo } & \multicolumn{2}{c}{ Onocha et al., Gupta et al., Tapondjou et al., Cavalli et al., } \\
& & & estudado & 1999 & 2002 & 2002 & 2004 \\
\hline 1 & 1011 & 1018 & a-terpineno & 1,24 & 56,0 & 63,6 & 37,6 & 9,7 \\
2 & 1022 & 1022 & p-cymeno & 4,83 & 15,5 & 19,5 & 50,0 & 16,2 \\
3 & 1240 & 1237 & (Z)-ascaridol & 87,0 & 0,1 & 6,2 & 3,5 & 41,8 \\
4 & 1250 & 1252 & Piperitone & 0,7 & - & - & - & - \\
5 & 1295 & 1301 & (E)-ascaridol & 5,04 & - & - & - & - \\
\hline- & - & - & Constituição total & 98,81 & 71,6 & 89,3 & 91,1 & 67,7 \\
\hline
\end{tabular}

${ }^{\mathrm{a}}$ Calculado. ${ }^{\mathrm{b}}$ Tabelado.

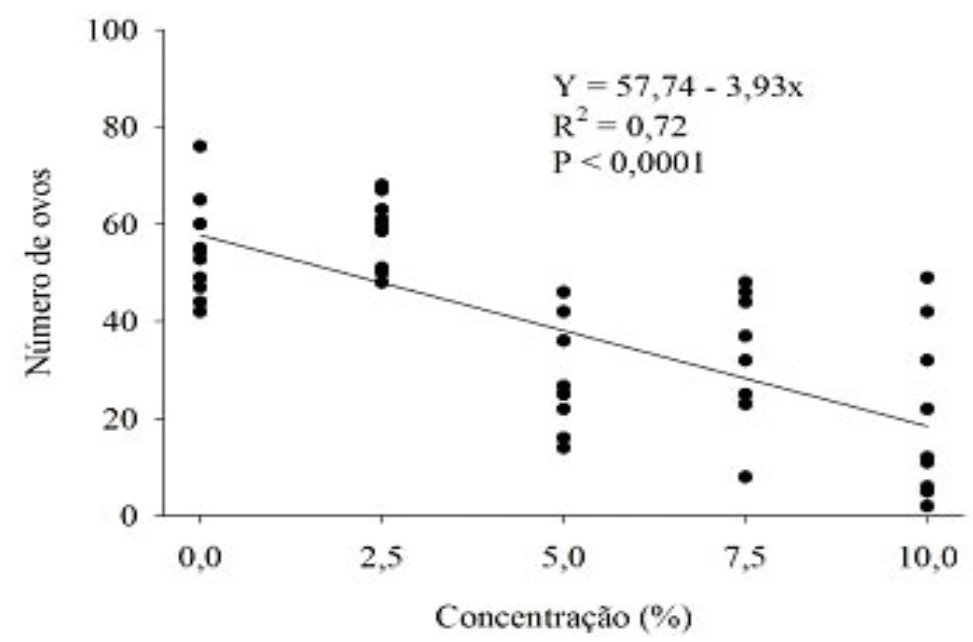

FIGURA 2 - Número de ovos depositados por Tetranychus urticae tratados com óleo essencial de ervade-santa-maria, Chenopodium ambrosioides, a diferentes concentrações ( $\left.\mathrm{v} \mathrm{v}^{-1}\right)$, após 24 h.

\section{CONCLUSÃO}

O ascaridol é o principal constituinte do óleo essencial de erva-de-santa-maria utilizado no presente trabalho.

A mortalidade do ácaro-rajado na concentração de $10 \%$ do óleo essencial de erva-de-santa-maria é significativa.

O óleo essencial de erva-de-santa-maria reduz significativamente a oviposição do ácaro-rajado.

O óleo essencial de erva-de-santa-maria mostra-se como uma opção promissora para o desenvolvimento de possíveis produtos fitossanitários para o manejo do ácaro-rajado.

\section{AGRADECIMENTOS}

À Coordenação de Aperfeiçoamento de Pessoal de Nível Superior (CAPES), pela concessão de bolsa de doutorado para Vando Rondelli, e ao Conselho Nacional de Desenvolvimento Científico e Tecnológico (CNPq), e pela concessão de bolsa de Pós-Doutorado para Ulysses Vianna. pela concessão de bolsa de Pós-Doutorado para Ulysses Vianna. 


\section{REFERÊNCIAS}

ADAMS, R.P. Identification of essential oil components by gas cromatography/mass spectroscopy. $4^{\text {th }}$ ed. Carol Stream: Allured Publishing Corporation, 2007. 804 p.

BOSTANIAN, N.J.; AKALACH, M.; CHIASSON, H. Effects of a Chenopodium-based botanical insecticide/acaricide on Orius insidiosus (Hemiptera: Anthocoridae) and Aphidius colemani (Hymenoptera: Braconidae). Pest Management Science, West Sussex, v.61, n.10, p.979-984, 2005.

BRITO, H.M.; GONDIM JR., M.G.C.; OLIVEIRA, J.V. de; CÂMARA, C.A.G. Toxicidade de formulações de neem (Azadirachta indica A. Juss.) ao ácaro-rajado e a Euseius alatus de leon e Phytoseiulus macropilis (Banks) (Acari: Phytoseiidae). Neotropical Entomology, São Paulo, v.35, n.4, p.500-505, 2006.

BURT, S. Essential oils: their antibacterial properties and potential applications in foods: a review. International Journal of Food Microbiology, Utrecht, v.94, n.3, p.223-253, 2004.

CALMASUR, O.; ASLAN, I.; SAHIN, F. Insecticidal and acaricidal effect of three Lamiaceae plant essential oils against Tetranychus urticae Koch and Bemisia tabaci Genn. Industrial Crops and Products, Erzurum, v.23, n.2, p.140-146, 2006.

CASTRO, H.G.; OLIVEIRA, L.O.; BARBOSA, L.C.A.; FERREIRA, F.A.; SILVA, D.J.H.; MOSQUIM, P.R.; NASCIMENTO, E.A. Teor e composição do óleo essencial de cinco acessos de mentrasto. Química Nova, São Paulo, v.27, n.1, p.55-57, 2004.

CAVALLI, J.F.; TOMI, F.; BERNARDINI, A.F.; CASANOVA, J. Combined analysis of the essential oil of Chenopodium ambrosioides by GC, GCMS and 13C-RMN spectroscopy: quantitative determination of ascaridole, a heatsensitive compound. Phytochemical Analysis, Chichester, v.15, n.5, p.275-279, 2004.

CHIASSON, H.; BOSTANIAN, N.J.; VINCENT, C. Acaricidal properties of a Chenopodium-based botanical. Journal of Economic Entomology, College Park, v.97, n.4, p.1373-1377, 2004.
CLOYD, R.A.; CHIASSON, H. Activity of an essential oil derived from Chenopodium ambrosioides on greenhouse insect pests. Journal of Economic Entomology, College Park, v.100, n.2, p.459-466, 2007.

COSTA, A.V.; PINHEIRO, P.F.; RONDELLI, V. M.; QUEIROZ, V. T.; TULER, A. C.; BRITO, K. B.; STINGUEL, P.; PRATISSOLI, D. Cymbopogon citratus (Poaceae) ESSENTIALOIL ON Frankliniella schultzei (Thysanoptera: Thripidae) AND Myzus persicae (Hemiptera: Aphididae). Bioscience Journal, Uberlândia, v. 29, n.6, p. 1840-1847, 2013.

CRESPO, A.L.B.; PICANÇO, M.C.; BACCI, L.; PEREIRA, E.J.G.; GONRING, A.H.R. Seletividade fisiológica de inseticidas a Vespidae predadores de Ascia monuste orseis. Pesquisa Agropecuária Brasileira, Brasília, v.37, n.3, p.237-242, 2002.

DE-OLIVEIRA, A.C.; RIBEIRO-PINTO, L.F.; PAUM-GARTTEN, J.R. In vitro inhibition of CYP2B1 monooxygenase by beta-myrcene and other monoternoid compounds. Toxicology Letters, Amsterdam, v.92, n.1, p. 39-46, 1997.

FADINI, M.A.M.; PALLINI, A.; VENZON, M. Controle de ácaros em sistema de produção integrada de morango. Ciência Rural, Santa Maria, v.34, n.4, p.1271-1277, 2004.

FERLA, N.J.; MARCHETTI, M; JOHANN, L.; HAETINGER, C. Functional response of Phytoseiulus macropilis under different Tetranychus urticae (Acari: Phytoseiidae, Tetranychidae) population density in laboratory. Zoologia, Curitiba, v.28, n.1, p.17-22, 2011.

FERLA, N.J.; MARCHETTI, M.M.; GONÇALVES, D. Ácaros predadores (Acari) associados à cultura do morango (Fragaria sp, Rosaceae) e plantas próximas no Estado do Rio Grande do Sul. Biota Neotropica, Campinas, v.7, n.2, p 1-8, 2007.

GIRÃO FILHO, J.E.; ALCÂNTARA NETO, F.; PÁDUA, L.E.M.; PESSOA, E.F. Repelência e atividade inseticida de pós vegetais sobre Zabrotes subfasciatus Boheman em feijão-fava armazenado. Revista Brasileira de Plantas Medicinais, Campinas, v.16, n.3, p.499-504, 2014. 
GUPTA, D.; CHARLES, R.; MEHTA, V.K.; GARG, S.N.; KUMAR, S. Chemical examination of the essential oil of Chenopodium ambrosioides L. from the southern hills of India. Journal of Essential Oil Research, London, v.14, n.2, p.93- 94, 2002.

HAN, J.; KIM, S.; CHOI, B.R.; LEE, S.G.; AHN, Y.J. Fumigant toxicity of lemon eucalyptus oil constituents to acaricide-susceptible and acaricideresistant Tetranychus urticae. Pest Management Science, West Sussex, v.67, n.12, p.1583-1588, 2011.

HENZ, G.P. Desafios enfrentados por agricultores familiares na produção de morango no Distrito Federal. Horticultura Brasileira, Brasília, v.28, n.3, p.260-265, 2010.

JARAMILLO, C.B.E.; DUARTE, R.E.; DELGADO, W. Bioactividad del aceite esencial de Chenopodium ambrosioides colombiano. Revista Cubana de Plantas Medicinais, Havana, v.17, n.1, p. 54-64, 2012.

MIRESMAILLI, S.; BRADBURY, R.; ISMAN, M.B. Comparative toxicity of Rosmarinus officinalis L. essential oil and blends of its major constituents against Tetranychus urticae Koch (Acari: Tetranychidae) on two different host plants. Pest Management Science, West Sussex, v.62, n.2, p.366-371, 2006.

MONZOTE, L.; STAMBERG, W.; STANIEK, K.; GILLE, L. Toxic effects of carvacrol, caryophyllene oxide, and ascaridole from essential oil of Chenopodium ambrosioides on mitochondria. Toxicology and Applied Pharmacology, New York, v.240, n.3, p.337-347, 2009.

MORAES, G.J.; FLECHTMANN, C.H.W. Manual de acarologia: acarologia básica e ácaros de plantas cultivadas no Brasil. Ribeirão Preto: Holos, 2008. 308 p.

MUHAYIMANA, A.; CHALCHAT, J.C.; GARRY, R.P. Chemical composition of essential oils of Chenopodium ambrosioides L. from Rwanda. Journal of Essential Oil Research, London, v.10, n.6, p.690-692, 1998.
ONOCHA, P.A.; EKUNDAYO, O.; ERAMO, T.; LAAKSO, I. Essential oil constituents of Chenopodium ambrosioides L. leaves from Nigeria. Journal of Essential Oil Research, London, v.11, n.2, p.220-222, 1999.

PINHEIRO, P. F.; QUEIROZ, V. T.; RONDELLI, V. M.; COSTA, A. V.; MARCELINO, T. P., PRATISSOLI, D. Insecticidal activity of citronella grass essential oil on Frankliniella schultzei and Myzus persicae. Ciência e Agrotecnologia, Lavras, v. 37, n. 2, p. 138-144, 2013.

PONTES, W.J.T.; OLIVEIRA, J.C.S. de; CAMARA, C.A.G. da; LOPES, A.C.H.R.; GONDIM, M.G.C. JR; OLIVEIRA, J.V. de; SCHWARTZ, M.O.E. Composition and acaricidal activity of the resin's essential oil of Protium bahianum Daly against two spotted spider mite (Tetranychus urticae). Journal of Essential Oil Research, London, v.19, n.4, p.379383, 2007a.

PONTES, W.J.T.; OLIVEIRA, J.C.G. DA; CÂMARA, C.A.G. DA; LOPES, A.C.H.R.; GONDIM JÚNIOR; M.G.C.; OLIVEIRA, J.V. DE; BARROS, R.; SCHWARTZ, M.O.E. Chemical composition and acaricidal activity of the leaf and fruit essential oils of Protium heptaphyllum (Aubl.) Marchand (Burseraceae). Acta Amazonica, Manaus, v.37, n.1, p.103-110, 2007 b.

PROCÓPIO, S.O.; VENDRAMIM, J.D.; RIBEIRO JÚNIOR, J.I.; SANTOS, J.B. Bioatividade de diversos pós de origem vegetal em relação à Sitophilus zeamais Mots. (Coleoptera: Curculionidae). Ciência e Agrotecnologia, Lavras v.27, n.6, p.1231-1236, 2003 .

RONDELLI, V.M.; PRATISSOLI, D.; POLANCZYK, R.A.; MARQUES, E.J.; STURM, G.M.; TIBURCIO, M.O. Associação do óleo de mamona com Beauveria bassiana no controle da traça-das-crucíferas. Pesquisa Agropecuária Brasileira, Brasília, v.46, n.2, p. 212-214, 2011.

RYAN, M.F.; BYRNE, O. Plant insect coevolution and inhition of acetylcholinestrase. Journal of Chemical Ecology, New York, v.14, n.10, p.19651975, 1988. 
SATO, M.E.; SILVA, M.; GONÇALVES, L.R. Differential toxicity of pesticides to Neoseiulus californicus (McGregor) (Acari: Phytoseiidae) and Tetranychus urticae Koch (Acari: Tetranychidae) on strawberry. Neotropical Entomology, São Paulo, v.31, n.3, p.449-456, 2002.

SCHLESENER, D.C.H; DUARTE, A.F.; GUERRERO, M.F.C; CUNHA, U.S. da; NAVA, D.E. Efeitos do nim sobre Tetranychus urticae Koch (Acari: Tetranychidae) e os predadores Phytoseiulus macropilis (Banks) e Neoseiulus californicus (Mcgregor) (Acari: Phytoseiidae). Revista Brasileira de Fruticultura, Jaboticabal, v.35, n.1, p.059-066, 2013.

SHI, G.L.; ZHAO, L.L.; LIU, S.Q.; CAO, H.; CLARKE, S.R.; SUN, J.H. Acaricidal activities of extracts of Kochia scoparia against Tetranychus urticae, Tetranychus cinnabarinus, and Tetranychus viennensis (Acari: Tetranychidae). Journal of Economic Entomology, London, v.99, n.3, p.858863, 2006.

TAPONDJOU, L.A.; ADLER, C.; BOUDA, H.; FONTEM, D.A. Efficacy of powder and essential oil from Chenopodium ambrosioides leaves as postharvest grain protectants against six-stored product beetles. Journal of Stored Products Research, Elmsford, v. 38, n.4, p.395-402, 2002.
TAVARES, M.A.G.C.; VENDRAMIM, J.D. Bioatividade da ervade-santa-maria, Chenopodium ambrosioides L., sobre Sitophilus zeamais Mots. (Coleoptera: Curculionidae). Neotropical Entomology, São Paulo, v.34, n.2, p.319-323, 2005.

TSUKAMOTO. Y.; NAKAGAWA, H.; KAJINO, H.; SATO, K.; TANAKA, K.; YANAI. T. Synthesis of novel 25-substituted milbemycin A4 derivatives and their acaricidal activity against Tetranychus urticae. Bioscience, Biotechnology and Biochemistry, Tokyo v.61, n.10, p.1650-1657, 1997.

VIEIRA, M.R.; SACRAMENTO, L.V.S.; FURLAN, L.O.; FIGUEIRA, J.C.; ROCHA, A.B.O. Efeito acaricida de extratos vegetais sobre fêmeas de Tetranychus urticae Koch (Acari: Tetranychidae). Revista Brasileira de Plantas Medicinais, Botucatu, v.8, n.4, p.210-217, 2006.

WEKESA, V.W.; VITAL, S.; SILVA, R.A.; ORTEGA, E.M.M.; KLINGEN, I.; DELALIBERA JR, I. The effect of host plants on Tetranychus evansi, Tetranychus urticae (Acari: Tetranychidae) and on their fungal pathogen Neozygites floridana (Entomophthorales: Neozygitaceae). Journal of Invertebrate Pathology, San Diego, v.107, n.2, p.139-145, 2011. 